\title{
Evaluación de los conocimientos, actitudes y prácticas de los estudiantes de escuelas vocacionales de servicios de salud respecto a COVID-19
}

\author{
Serhat Sirekbasan, ${ }^{1 *}$ Aysegul Oglakci Ilhan ${ }^{1}$ y Canan Baydemir ${ }^{2}$ \\ 'Universidad Çankırı Karatekin, Escuela Vocacional de Servicios de Salud de Eldivan, Departamento de Técnicas de Laboratorio Médico, Çankırı, \\ Turquía; ${ }^{2}$ Universidad Kocaeli, Facultad de Medicina, Departamento de Bioestadística e Informática Médica, Kocaeli, Turquía
}

\section{Resumen}

Introducción: La intervención más crítica para controlar la infección por SARS-CoV-2 requiere el conocimiento y la actitud correctos hacia el virus. Objetivo: Determinar el conocimiento, las actitudes y las prácticas de los estudiantes de una escuela vocacional de servicios de salud respecto a COVID-19. Métodos: Estudio transversal realizado entre mayo y junio de 2020 en una universidad de la región de Turquía. Los datos se recopilaron mediante un portal de encuestas en línea. Resultados: Se completaron y devolvieron 454 cuestionarios. Los estudiantes de técnicas de laboratorio médico y primeros auxilios y emergencias tuvieron puntuaciones de conocimiento significativamente más altas $(p<0.001)$. Más de $43 \%$ de los estudiantes $(n=201)$ usaron televisores como su principal fuente de información sobre COVID-19. Se manifestó una actitud positiva hacia las aplicaciones, así como evitar lugares concurridos y mantener la higiene de las manos y la distancia social. Conclusiones: Los estudiantes de la escuela vocacional de servicios de salud tienen buen conocimiento y actitud positiva hacia COVID-19. Las principales fuentes de información fueron la televisión y las redes sociales, por lo que se debe prestar más atención a estos temas en el plan de estudios para utilizar fuentes de información basadas en la ciencia.

PALABRAS CLAVE: Actitud. Coronavirus. COVID-19. Conocimiento. SARS-COV-2.

\section{Evaluation of knowledge, attitudes and practices of health services vocational schools' students with regard to COVID-19}

\begin{abstract}
Introduction: The most critical intervention to control SARS-CoV-2 infection requires appropriate knowledge and attitude towards the virus. Objective: To determine the level of knowledge, attitudes and practices of students of a health services' vocational school with regard to COVID-19. Methods: Cross-sectional study carried out between May and June 2020 in a university of the Turkish region. Data were collected through an online survey portal. Results: Four hundred and fifty-four questionnaires were completed and returned. Students of medical laboratory techniques and first aid and emergency medicine had significantly higher knowledge scores $(p<0.001)$. More than $43 \%$ of the students $(n=201)$ used the TV as their main source of information on COVID-19. There was a positive attitude towards preventive measures such as avoiding crowded places and maintaining hand hygiene and social distance. Conclusions: Health services vocational school students have good knowledge and positive attitude towards COVID-19. The main sources of information were the TV and social media. For this reason, more attention should be given to these issues in the curriculum in order for science-based information sources to be used.
\end{abstract}

KEY WORDS: Attitude. Coronavirus. COVID-19. Knowledge. SARS-CoV-2.

Correspondencia:

*Serhat Sirekbasan

E-mail: serhatsirekbasan@gmail.com
Gac Med Mex. 2021;157:74-79

Disponible en PubMed

www.gacetamedicademexico.com

0016-3813/๑ 2020 Academia Nacional de Medicina de México, A.C. Publicado por Permanyer. Este es un artículo open access bajo la licencia CC BY-NC-ND (http://creativecommons.org/licenses/by-nc-nd/4.0/). 


\section{Introducción}

Los coronavirus se han identificado desde hace décadas y generalmente son patógenos respiratorios relativamente inofensivos. Sin embargo, después de la aparición del síndrome respiratorio agudo severo (SARS), en 2002, y del síndrome respiratorio del Medio Oriente (MERS), en 2017, han llamado la atención como patógenos importantes en las infecciones respiratorias. ${ }^{1}$ Se determinó que un miembro de esta familia de virus causó la epidemia que surgió en Wuhan, China, en diciembre de 2019 y se convirtió en una amenaza global que afecta a todo el mundo. ${ }^{2,3}$

La enfermedad del coronavirus 2019, también conocida como COVID-19, es causada por un nuevo coronavirus humano llamado SARS-CoV-2 y se ha propagado rápidamente por todo el mundo. Al 25 de junio de 2020, en el mundo se informaron más de 9.2 millones de casos confirmados y 478691 muertes. ${ }^{4}$ COVID-19 se transmite de persona a persona a través de gotitas, por contacto oral con residuos fecales contaminados y por el contacto directo con una persona infectada. ${ }^{5}$ Hasta la fecha no hay tratamientos curativos antivirales o vacunas recomendadas contra COVID-19, que tiene un periodo de incubación de dos a 14 días. ${ }^{6,7}$ Existe una fuerte relación entre el aumento de la gravedad de la enfermedad y la mortalidad en personas de edad avanzada y pacientes con comorbilidades como hipertensión, enfermedad cardiaca, enfermedad pulmonar, cáncer o diabetes. La enfermedad es altamente contagiosa y sus principales síntomas clínicos son fiebre, tos seca, fatiga, dolor muscular y disnea. ${ }^{8,9}$

La intervención más crítica para controlar la infección por SARS-CoV-2 es aplicar medidas preventivas comunes que se utilizan para evitar la infección por otros virus respiratorios. El distanciamiento social es la primera de ellas. Las personas deben evitar el contacto con personas infectadas, especialmente debido a la naturaleza infecciosa del virus. También es importante que las personas se laven las manos con agua y jabón durante al menos 30 segundos 0 , en ausencia de estos, que usen desinfectantes para manos. ${ }^{10-12}$

El conocimiento y las actitudes de los estudiantes de las escuelas vocacionales de servicios de salud, quienes formarán parte del sistema de salud en el futuro, son muy importantes en términos de prevención de infecciones. Durante los posibles periodos epidémicos, mostrar una actitud positiva hacia la prevención de la transmisión de agentes infecciosos brinda una oportunidad única para combatir las enfermedades. Por lo tanto, el objetivo de nuestro estudio es determinar los niveles de conocimiento, las actitudes respecto a COVID-19 y evaluar el comportamiento de dichos estudiantes. Nuestro estudio es el primero relativo al tema.

\section{Métodos}

Estudio transversal realizado entre mayo y junio de 2020 en una universidad de la región de Anatolia Central de Turquía. La investigación examinó a los estudiantes de los departamentos vocacionales de servicios de salud de la universidad. La muestra estuvo compuesta por los individuos que aceptaron participar voluntariamente.

Se desarrolló una encuesta en línea para la investigación, cuya validez y confiabilidad se evaluó antes de su uso. En el primer paso se utilizaron opiniones de expertos para garantizar la validez del contenido de las declaraciones en el grupo de preguntas. En el segundo paso se realizó un estudio piloto con 11 estudiantes para validar el cuestionario; los resultados fueron consistentes.

Los datos de la investigación se recopilaron a través de Microsoft Forms (https://forms.office.com/), un portal de encuestas en línea. Se compartió el enlace de la encuesta con los grupos de estudiantes mediante WhatsApp y se envió una invitación para participar. En la primera página de la encuesta se informó a los estudiantes sobre el estudio y la participación voluntaria. La encuesta está constituida por 31 preguntas, cuya contestación requiere aproximadamente seis minutos.

La encuesta contiene cuatro secciones sobre conciencia (conocimiento) y actitud respecto a la pandemia de COVID-19. La primera sección incluye las características de los participantes (edad, sexo y el programa en el que están estudiando), la segunda incluye preguntas en torno a la conciencia sobre COVID-19 (15 preguntas), la tercera se refiere a fuentes de información sobre COVID-19 (una pregunta) y la cuarta contiene preguntas sobre actitudes y prácticas (10 preguntas).

Las evaluaciones estadísticas se realizaron con el programa IBM SPSS versión 20.0 (IBM Corp., Armonk, NY, Estados Unidos). La conformidad de los datos con una distribución normal se evaluó mediante la prueba de Shapiro-Wilk. Las variables numéricas se describen como medianas (percentiles 25-75) y frecuencias (porcentajes). Respecto a las variables 
Tabla 1. Características demográficas y puntuación de conocimiento de los participantes

\begin{tabular}{|c|c|c|c|c|c|c|}
\hline \multirow[t]{2}{*}{ Características } & \multicolumn{2}{|c|}{ Participantes } & \multicolumn{4}{|c|}{ Puntuación de conocimiento } \\
\hline & $\mathrm{n}$ & $\%$ & Mediana & Media & DE & $p$ \\
\hline $\begin{array}{l}\text { Sexo } \\
\text { Femenino } \\
\text { Masculino }\end{array}$ & $\begin{array}{l}316 \\
138\end{array}$ & $\begin{array}{l}69.6 \\
30.4\end{array}$ & $\begin{array}{l}12.80 \\
12.68\end{array}$ & $\begin{array}{l}13 \\
13\end{array}$ & $\begin{array}{l}1.02 \\
1.12\end{array}$ & $0.373^{*}$ \\
\hline $\begin{array}{l}\text { Grupo de edad (años) } \\
\leq 18 \\
19-21 \\
22-24 \\
\geq 25\end{array}$ & $\begin{array}{c}22 \\
373 \\
48 \\
11\end{array}$ & $\begin{array}{c}4.8 \\
82.2 \\
10.6 \\
2.4\end{array}$ & $\begin{array}{l}12.64 \\
12.72 \\
13.06 \\
13.09\end{array}$ & $\begin{array}{l}13 \\
13 \\
13 \\
13\end{array}$ & $\begin{array}{l}0.90 \\
1.07 \\
0.93 \\
0.94\end{array}$ & $0.100^{\dagger}$ \\
\hline $\begin{array}{l}\text { Programa } \\
\text { Desarrollo Infantil } \\
\text { Cuidado de los Ancianos } \\
\text { Salud Ambiental } \\
\text { Primeros Auxilios y Emergencias } \\
\text { Atención Domiciliaria del Paciente } \\
\text { Laboratorio y Salud Veterinaria } \\
\text { Técnicas de Laboratorio Médico } \\
\text { Óptico }\end{array}$ & $\begin{array}{c}69 \\
19 \\
32 \\
152 \\
90 \\
29 \\
30 \\
33\end{array}$ & $\begin{array}{c}15.2 \\
4.2 \\
7.0 \\
33.5 \\
19.8 \\
6.4 \\
6.6 \\
7.3\end{array}$ & $\begin{array}{l}12.33 \\
13.00 \\
12.41 \\
13.08 \\
12.51 \\
12.55 \\
13.40 \\
12.73\end{array}$ & $\begin{array}{c}12 \\
13 \\
12.5 \\
13 \\
13 \\
13 \\
13 \\
13\end{array}$ & $\begin{array}{l}0.98 \\
1.05 \\
1.04 \\
0.92 \\
1.08 \\
1.15 \\
0.81 \\
1.13\end{array}$ & $0.000^{+}$ \\
\hline
\end{tabular}

numéricas que no tenían distribución normal, la diferencia entre los grupos o materiales se comparó mediante la prueba $\mathrm{U}$ de Mann-Whitney; en las variables categóricas se utilizaron las pruebas de chi cuadrada de Fisher, de Yates y de Monte Carlo. El nivel de significación estadística se estableció en $p<0.05$ (dos lados).

El estudio fue aprobado por el Comité de Ética de la Universidad Çankırı Karatekin (número de aprobación 2020/210) y se obtuvo un permiso de la dirección de la escuela (2020/E.9228); se realizó de acuerdo con los principios de la Declaración de Helsinki. En la primera página del cuestionario se mostró un formulario electrónico de consentimiento voluntario informado.

\section{Resultados}

Un total de 460 estudiantes completaron el cuestionario, de los cuales se excluyeron seis que se negaron a aceptar el formulario electrónico de consentimiento voluntario informado. En la muestra final $(n=454)$, la mayoría de los participantes tenía entre 19 y 21 años $(n=373,82.2 \%)$. Las mujeres representaron $69.6 \%$ de la muestra $(n=316)$. Otras características demográficas se describen en la Tabla 1.

En la Tabla 2 se muestra el estado actual de la información sobre COVID-19 entre los estudiantes universitarios vocacionales de salud. El nivel de conocimiento se evaluó con el otorgamiento de un punto para las respuestas correctas y de cero para las incorrectas. La puntuación más alta fue de 15 y la puntuación más baja, de nueve. Un total de 401 (88.3\%) participantes obtuvieron buenas puntuaciones, pero $53(11.7 \%)$ mostraron información insuficiente sobre COVID-19.

La mayoría de los encuestados tenía información sobre las medidas de prevención de infecciones, los factores de riesgo de la enfermedad y que el contacto cercano es efectivo para propagar la enfermedad. Inesperadamente, la mayoría de los encuestados creía que el periodo de incubación del virus era mucho más largo de lo que realmente es. Además, la información débil fue más evidente en las preguntas sobre el lugar de los antibióticos en el tratamiento de primera línea y la efectividad del alcohol a $70 \%$, para lo cual las tasas de respuesta incorrecta fueron 38.1 y $33.3 \%$, respectivamente. La puntuación promedio de conocimiento fue $12.76 \pm 1.05$ (Tabla 2).

Las puntuaciones promedio de conocimiento total se compararon de acuerdo con las características demográficas de los estudiantes (Tabla 1). No hubo diferencias estadísticamente significativas conforme el grupo de edad ni el sexo ( $p>0.05)$. Según los programas de estudio, los participantes que estudiaban técnicas de laboratorio médico y primeros auxilios y emergencias obtuvieron puntuaciones de 
Tabla 2. Conocimiento de estudiantes de escuelas vocacionales de salud sobre COVID-19

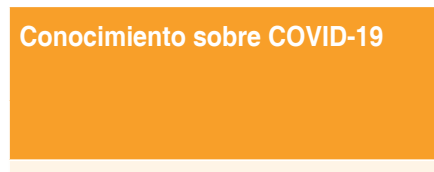

Las personas mayores y las personas con problemas médicos subyacentes como hipertensión, diabetes, corazón y cáncer tienen más probabilidades de desarrollar enfermedades graves

El aislamiento y el tratamiento de personas infectadas con el virus es una forma efectiva de reducir la propagación de la enfermedad

Las personas que contactan a una persona infectada deben aislarse de inmediato durante 14 días en un lugar conveniente

Lavarse las manos con agua y jabón durante al menos 20 segundos puede ayudar a prevenir infecciones

Se desarrolla enfermedad respiratoria aguda grave en pacientes con COVID-19

La principal fuente de COVID-19 es una planta

Fiebre, tos seca y dificultad para respirar son síntomas distintivos de COVID-19

La enfermedad puede transmitirse desde pacientes asintomáticos o en el periodo latente de la enfermedad

Las personas con COVID-2019 no pueden infectar a otros cuando no hay fiebre

No es necesario que los niños y adultos jóvenes tomen medidas para prevenir la transmisión de COVID-19

COVID-19 puede transmitirse por picadura de mosca

\section{La reacción en cadena de la} polimerasa (PCR) se puede utilizar para el diagnóstico de COVID-19

COVID-19 se puede eliminar con alcohol a $70 \%$

Los antibióticos son el tratamiento de primera línea

El periodo de incubación del virus es de 2 a 4 semanas

El conocimiento se evaluó con el otorgamiento de un punto a las respuestas correctas y de cero, a las incorrectas. Los valores correctos de información de respuesta se midieron en una escala con un máximo de 15 y un mínimo de cero. La puntuación promedio de conocimiento fue $12.76 \pm 1.05$.

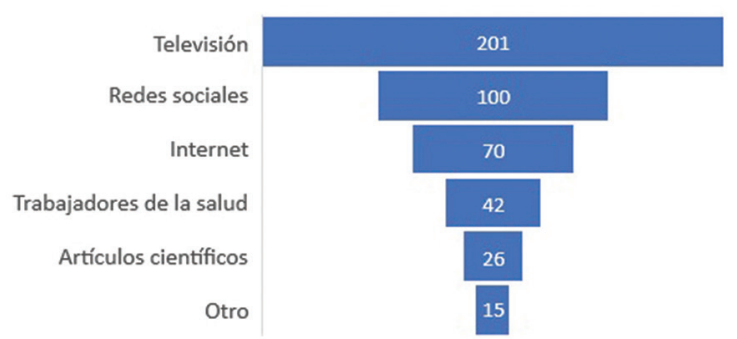

Figura 1. Fuentes de información sobre COVID-19 en 454 participantes.

conocimiento total más altas, con diferencia estadísticamente significativa entre ellos ( $p<0.001)$. Los participantes que tomaron cursos de microbiología a lo largo de su educación obtuvieron puntuaciones más altas en la escala de conocimiento (12.87) que otros estudiantes (12.69), pero la diferencia no fue estadísticamente significativa $(p=0.071)$.

Las respuestas mostraron que los estudiantes accedían principalmente a la información de la televisión (Figura 1). La mayoría de los participantes $(90.4 \%)$ no había asistido a lugares concurridos en los últimos días y usaba máscaras al salir de la casa $(95.0 \%)$. A pesar de esto, un pequeño segmento recientemente había visitado lugares concurridos $(9.6 \%)$ y no usaba máscara al salir de casa $(5.0 \%)$.

En la sección del cuestionario relativa a actitud se identificó que existía una actitud positiva hacia prácticas tales como frecuente higiene ambiental y de manos, mantener la distancia social y evitar lugares concurridos. Los participantes creían que incluso las personas sanas deberían usar máscaras y que se deberían tomar medidas adicionales para prevenir la transmisión de la enfermedad. La mayoría de los participantes creía que el peligro de COVID-19 no es tanto como el de la gripe estacional. Sin embargo, la mitad de los participantes consideraba que no todas las personas con COVID-19 desarrollan afección grave (Tabla 3).

\section{$332 \quad 73.1 \quad 122 \quad 26.9 \quad$ Discusión}

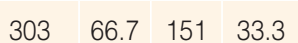

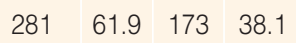
\begin{tabular}{l|l|l|l}
66 & 14.5 & 388 & 85.5
\end{tabular}

(5)

Muchos estudios han examinado una variedad de información, actitudes y prácticas sobre las enfermedades epidémicas infecciosas causadas por coronavirus como SARS y MERS. Sin embargo, hasta donde sabemos, no hay investigaciones que examinen el conocimiento y las actitudes de los estudiantes de escuelas vocacionales de servicios de salud respecto a COVID-19. Los resultados de este estudio mostraron que estos estudiantes tienen buenos conocimientos y 
Tabla 3. Actitud de los estudiantes de educación vocacional de salud hacia COVID-19

\begin{tabular}{|c|c|c|c|c|c|c|c|c|c|}
\hline \multirow[t]{3}{*}{ Actitudes } & \multicolumn{6}{|c|}{ Respuestas } & \multicolumn{3}{|c|}{$\mathbf{p}^{\dagger}$} \\
\hline & \multicolumn{2}{|c|}{ A } & \multicolumn{2}{|c|}{$\mathbf{U}$} & \multicolumn{2}{|c|}{ D } & \multirow[t]{2}{*}{ Programa } & \multirow[t]{2}{*}{ Sexo } & \multirow[t]{2}{*}{ Años } \\
\hline & $\mathrm{n}$ & $\%$ & $n$ & $\%$ & $n$ & $\%$ & & & \\
\hline $\begin{array}{l}\text { Las personas deben evitar ir a lugares con mucha gente para } \\
\text { prevenir el contagio por COVID- } 19\end{array}$ & 452 & 99.6 & 0 & 0 & 2 & 0.4 & 0.409 & 0.516 & 1.000 \\
\hline $\begin{array}{l}\text { Incluso si no tenemos signos de enfermedad, debemos mantener } \\
\text { nuestra distancia social }\end{array}$ & 454 & 100 & 0 & 0 & 0 & 0 & - & - & - \\
\hline $\begin{array}{l}\text { Debemos limpiar las superficies de uso frecuente como manijas de } \\
\text { puertas, grifos, fregaderos todos los días con agua y detergente }\end{array}$ & 446 & 98.2 & 7 & 1.5 & 1 & 0.2 & 0.030 & 0.084 & 0.616 \\
\hline $\begin{array}{l}\text { Definitivamente debemos lavarnos las manos después del contacto } \\
\text { con superficies potencialmente sucias }\end{array}$ & 454 & 100 & 0 & 0 & 0 & 0 & - & - & - \\
\hline Una persona sana no necesita usar una máscara & 15 & 3.3 & 4 & 0.9 & 435 & 95.8 & 0.067 & 0.100 & 0.084 \\
\hline $\begin{array}{l}\text { No se desarrollará una enfermedad grave en cada individuo con } \\
\text { COVID-19 }\end{array}$ & 187 & 41.2 & 90 & 19.8 & 177 & 39.0 & 0.011 & 0.552 & 0.249 \\
\hline El peligro de COVID-19 es tanto como el de la gripe estacional & 13 & 2.9 & 52 & 11.5 & 389 & 85.7 & 0.073 & 0.410 & 0.081 \\
\hline $\begin{array}{l}\text { No necesito tomar medidas adicionales para prevenir el contagio } \\
\text { por COVID-19 }\end{array}$ & 4 & 0.9 & 9 & 2.0 & 441 & 97.1 & 0.055 & 0.641 & 0.300 \\
\hline
\end{tabular}

una actitud positiva hacia COVID-19. La mayoría era consciente de la condición de la enfermedad, que continúa a nivel de pandemia en todo el mundo.

Se concluyó que las variables de sexo y grupo de edad no afectaron las puntuaciones promedio totales. En un artículo de revisión sistemática de 2013, Tooher et al. ${ }^{13}$ examinaron el conocimiento, el comportamiento y las actitudes de la comunidad respecto a la epidemia de gripe H1N1. Sus hallazgos respaldan nuestros resultados, si bien otras investigaciones han informado que el sexo y el grupo de edad afectan el conocimiento y la conciencia que se tiene sobre la pandemia. Se cree que estas diferencias entre los estudios pueden deberse a la variabilidad de los países donde se realizaron, el factor que causa la pandemia y la población incluida.

Además, los participantes que estudiaban técnicas de laboratorio médico y primeros auxilios y programas de emergencia lograron mejores puntuaciones de conocimiento sobre COVID-19 que los estudiantes de otros programas. Esta diferencia puede explicarse por el hecho de que los primeros tienen más conocimiento clínico porque están en contacto directo con los pacientes, tanto en sus cursos como en sus aplicaciones en instituciones de salud.

Identificamos que la televisión fue la principal fuente de información sobre COVID-19 en más de $43 \%$ de los estudiantes $(n=201)$. Este hallazgo es consistente con el de otra investigación en estudiantes, quienes eligieron medios de comunicación masiva para obtener información general sobre SARS..$^{14}$ Sin embargo, es una preocupación importante que más de $21 \%$ de los estudiantes $(n=100)$ utilice las redes sociales como su principal fuente de información. Es muy importante obtener información imparcial y confiable sobre la infección por COVID-19.

Actualmente, los científicos expertos en su campo aparecen con frecuencia en programas de televisión y se comparte conocimiento valioso sobre la prevención y el control de la pandemia actual. Sin embargo, existe una amplia gama de información maliciosa, especialmente en las redes sociales, que se está extendiendo rápidamente y que puede llevar a error a las personas. Por lo tanto, la información sobre COVID-19 debe evaluarse cuidadosamente y usar como fuentes los medios con contenido científico.

Los resultados de este estudio muestran que parte de la información disponible sobre COVID-19 es incorrecta e incompleta. Muchos participantes no sabían sobre el periodo de incubación (85.5 \%) y el lugar de los antibióticos en el tratamiento de primera línea (38.1\%). También hubo importantes deficiencias acerca del conocimiento de la efectividad del alcohol a $70 \%(33.3 \%)$ y la transmisión a través de las picaduras de mosquitos (18.5\%). Las infecciones respiratorias han sido la causa más común de prescripción de antibióticos en el servicio de atención primaria durante muchos años, ${ }^{15}$ lo cual resulta lamentable 
porque los antibióticos se recetan inapropiadamente a pesar de la ausencia de infección bacteriana comprobada y del creciente conocimiento de que puede haber soluciones para las infecciones causadas por virus en toda la comunidad. Por esta razón, necesitamos aumentar nuestra conciencia como sociedad sobre el uso correcto de los antibióticos. También es importante fomentar el desarrollo del conocimiento en los estudiantes de los departamentos relacionados con la salud, quienes pueden desempeñar un papel activo en la prevención de tales epidemias en el futuro y la enfermedad actual, que sigue teniendo un efecto destructivo global. Según lo sugerido por Drake et al., ${ }^{16}$ existe la necesidad de un método de aprendizaje social con el que la carga de las infecciones con riesgo de pandemia global pueda reducirse efectivamente.

\section{Conclusiones}

Según los resultados de nuestro estudio, se determinó que los estudiantes de una escuela vocacional de salud tienen buenos conocimientos y una actitud positiva hacia COVID-19. Aunque los participantes manifestaron información suficiente, todavía existen deficiencias e inexactitudes en algunos aspectos. Se conocen condiciones como los factores de riesgo de la enfermedad y el efecto del contacto cercano sobre la propagación de la enfermedad, y la mayoría de los participantes aplica medidas de prevención de infecciones. Las principales fuentes de información para los estudiantes fueron la televisión y las redes sociales. En línea con estos resultados se puede considerar el desarrollo de nuevas políticas para alentar a los estudiantes a emplear recursos de información basados en la ciencia.

\section{Conflicto de intereses}

Ninguno.

\section{Financiamiento}

Esta investigación no recibió ninguna subvención específica de agencias del sector público o comercial o entidades sin fines de lucro.

\section{Responsabilidades éticas}

Protección de sujetos humanos y animales. Los autores declaran que no realizaron experimentos en humanos o animales para este estudio.

Confidencialidad de los datos. Los autores declaran que en este artículo no aparecen datos de pacientes.

Derecho a la privacidad y consentimiento informado. Los autores declaran que en este artículo no aparecen datos de pacientes.

\section{Bibliografía}

1. Yin $Y$, Wunderink RG. MERS, SARS and other coronaviruses as causes of pneumonia. Respirology. 2018;23:130-137.

2. Zhu N, Zhang D, Wang W, Li X, Yang B, Song J, et al. A novel coronavirus from patients with pneumonia in China, 2019. N Engl J Med. 2020;382:727-733

3. Hasöksüz M, Kiliç S, Saraç F. Coronaviruses and SARS-COV-2. Turk J Med Sci. 2020;50(SI-1):549-556.

4. World Health Organization [Internet]. Coronavirus disease 2019 (COVID-19) pandemic; 2020.

5. Li Q, Guan X, Wu P, Wang X, Zhou L, Tong Y, et al. Early transmission dynamics in Wuhan, China, of novel coronavirus-infected pneumonia. $\mathrm{N}$ Engl J Med. 2020;382:1199-1207.

6. Backer JA, Klinkenberg D, Wallinga J. Incubation period of 2019 novel coronavirus (2019-nCoV) infections among travellers from Wuhan, China, 20-28 January 2020. Euro Surveill. 2020;25:2000062.

7. Cascella M, Rajnik M, Cuomo A, Dulebohn SC, Di Napoli R. Features, evaluation and treatment coronavirus. EE. UU.: StatPearls Publishing; 2020.

8. Wang X, Fang X, Cai Z, Wu X, Gao X, Min J, et al. Comorbid chronic diseases and acute organ injuries are strongly correlated with disease severity and mortality among COVID-19 patients: a systemic review and meta-analysis. Research. 2020;2020:2402961.

9. Ashour HM, Elkhatib WF, Rahman MM, Elshabrawy HA. Insights into the recent 2019 novel coronavirus (SARS-CoV-2) in light of past human coronavirus outbreaks. Pathogens. 2020;9:186.

10. Bhagavathula AS, Aldhaleei WA, Rahmani J, Mahabadi MA, Bandari DK. Knowledge and perceptions of COVID-19 among health care workers: cross-sectional study. JMIR Public Health Surveill. 2020;6:e19160.

11. Conley L, Tao Y, Henry A, Koepf E, Cecchini D, Pieracci J, et al. Evaluation of eco-friendly zwitterionic detergents for enveloped virus inactivation. Biotechnol Bioeng. 2017;114:813-820.

12. Kampf G. Efficacy of ethanol against viruses in hand disinfection. J Hosp Infect. 2018:98:331-338.

13. Tooher R, Collins JE, Street JM, Braunack-Mayer A, Marshall H. Community knowledge, behaviours and attitudes about the $2009 \mathrm{H} 1 \mathrm{~N} 1$ influenza pandemic: a systematic review. Influenza Other Respir Viruses. 2013;7:1316-1327.

14. Bergeron SL, Sánchez AL. Media effects on students during SARS outbreak. Emerg Infect Dis. 2005;11:732-734.

15. Harris AM, Hicks LA, Qaseem A, High Value Care Task Force of the American College of Physicians and for the Centers for Disease Control and Prevention. Appropriate antibiotic use for acute respiratory tract infection in adults: advice for high-value care from the American College of Physicians and the Centers for Disease Control and Prevention. Ann Intern Med. 2016;164:425ロ434.

16. Drake JM, Chew SK, Ma S. Societal learning in epidemics: intervention effectiveness during the 2003 SARS outbreak in Singapore. PLoS One. 2006;1:e20 